\title{
Segurança do doente na atençáo primária: perceçáo de profissionais de equipas de saúde da família
} Patient safety in primary health care: the perceptions of professionals working in family health teams Seguridad del paciente en atención primaria: percepción de los profesionales de los equipos de salud familiar

\author{
Helen Cristiny Teodoro Couto Ribeiro \\ iD https://orcid.org/0000-0001-9365-7228 \\ Rafaela Dias Pardini ${ }^{2}$ \\ iD https://orcid.org/0000-0003-0725-8656 \\ Joseane da Silva ${ }^{3}$ \\ (iD https://orcid.org/0000-0002-0053-5764 \\ Aline Carrilho Menezes ${ }^{1,4}$ \\ iD https://orcid.org/0000-0001-7658-4039 \\ Elaine Cristina Dias Franco ${ }^{1}$ \\ (iD https://orcid.org/0000-0001-8744-7726 \\ Luciana Regina Ferreira da Mata ${ }^{5}$ \\ iD https://orcid.org/0000-0002-5080-4643 \\ Cecília Godoi Campos ${ }^{6}$ \\ (iD https://orcid.org/0000-0002-0075-8909
}

${ }^{1}$ Universidade Federal de São João del-Rei, Divinópolis, Minas Gerais, Brasil.

${ }^{2}$ Universidade do Estado de Minas Gerais, Divinópolis, Minas Gerais, Brasil

${ }^{3}$ Secretaria Municipal de Saúde de Divinópolis, Divinópolis, Minas Gerais, Brasil

${ }^{4}$ Escola Técnica de Enfermagem do Complexo de Saúde São Joáo de Deus, Divinópolis, Minas Gerais, Brasil

${ }^{5}$ Universidade Federal de Minas Gerais, Belo Horizonte, Minas Gerais, Brasil

${ }^{6}$ Secretaria de Estado de Saúde de Minas Gerais, Divinópolis, Minas Gerais, Brasil

Autor de correspondência Helen Cristiny Teodoro Couto Ribeiro E-mail: helen.cristiny@ufsj.edu.br

Recebido: 02.06.20

Aceite: 24.02 .21

\section{Resumo}

Enquadramento: Considerando que muitos cuidados acontecem na atenção primária à saúde, é necessária uma melhor compreensão da segurança do doente neste nível de atenção.

Objetivo: Analisar a perceção de profissionais de saúde que atuam em equipas de saúde da família acerca da segurança do doente.

Metodologia: Estudo exploratório com abordagem qualitativa. Participaram 23 profissionais (enfermeiros, técnicos de enfermagem, médicos e agentes comunitários) de 6 equipas de estratégia de saúde da família no Brasil. Os dados foram recolhidos através de entrevistas com guiāo semiestruturado. Utilizou-se a técnica de análise de conteúdo temática de Bardin.

Resultados: Da análise dos dados emergiram duas categorias temáticas: (Des) Conhecimento sobre os princípios da segurança do doente e Atuação dos profissionais e estratégias para a promoçáo da segurança do doente

Conclusão: A segurança do doente na atenção primária à saúde ainda é um tema que apresenta fragilidade de conhecimento por parte dos profissionais do estudo. Os enfermeiros foram os únicos profissionais que destacaram a repercussão atual da temática.

Palavras-chave: segurança do paciente; estratégia saúde da família; equipe de assistência ao paciente; atenção primária à saúde

\section{Abstract}

Background: Given that a considerable amount of care is provided in primary health care settings, a better understanding of patient safety at this level of care is essential.

Objective: To explore the perceptions of patient safety among healthcare professionals working in family health teams.

Methodology: An exploratory study, using a qualitative approach, was carried out with 23 health professionals (nurses, nurse technicians, physicians, and community health agents) from 6 family health strategy teams in Brazil. Data were collected through semi-structured interviews and analyzed using Bardin's thematic content analysis method.

Results: Two thematic categories emerged from the data analysis: (Lack of) Knowledge about patient safety principles and Professionals' actions and strategies for promoting patient safety.

Conclusion: Patient safety in primary health care is an issue still poorly understood among the study participants. The nurses were the only professionals who highlighted its current impact.

Keywords: patient safety; family health strategy; patient care team; primary health care

\section{Resumen}

Marco contextual: Considerando que gran parte de los cuidados se realiza en la atención primaria, se necesita una mejor comprensión de la seguridad del paciente en este nivel de atención.

Objetivo: Analizar la percepción de los profesionales sanitarios que trabajan en los equipos de salud familiar sobre la seguridad del paciente.

Metodología: Estudio exploratorio con enfoque cualitativo. Participaron 23 profesionales (enfermeros, técnicos de enfermería, médicos y trabajadores comunitarios) de 6 equipos de estrategia de salud familiar de Brasil. Los datos se recogieron mediante entrevistas con un guion semiestructurado. Se utilizó la técnica de análisis de contenido temático de Bardin.

Resultados: Del análisis de los datos surgieron dos categorías temáticas: (Des)conocimiento de los principios de la seguridad del paciente y Actuación de los profesionales y estrategias para la promoción de la seguridad del paciente.

Conclusión: La seguridad del paciente en la atención primaria sigue siendo un tema que presenta cierta fragilidad de conocimiento por parte de los profesionales del estudio. Los enfermeros fueron los únicos profesionales que destacaron la repercusión actual del tema.

Palabras clave: seguridad del paciente; estrategia de salud familiar; grupo de atención al paciente; atención primaria de salud

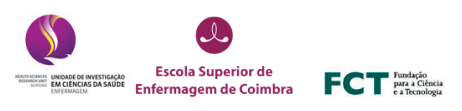

Como citar este artigo: Ribeiro, H. C., Pardini, R. D., Silva, J., Menezes, A. C., Franco, E. C., Mata, L. R., \& Campos, C. G. (2021). Segurança do doente na atenção primária: perceçăo de profissionais de equipas de saúde da família. Revista de Enfermagem Referência, 5(6), e20082. https://doi.org/10.12707/RV20082 


\section{Introdução}

A segurança do doente tornou-se uma das prioridades de discussão em todos os níveis de atenção à saúde. É fundamental prestar assistência isenta de riscos ao paciente, eficaz, eficiente e resolutiva. Os estudos com enfoque nesta temática, na sua maioria, são realizados em contexto hospitalar (Marchon et al., 2015). No entanto, é na atenção primária à saúde (APS) que se concentra grande parte dos cuidados realizados à população, desde açóes individuais e coletivas relacionadas com a promoção e proteção da saúde, prevenção de agravamento do estado de saúde até ao diagnóstico, tratamento e reabilitação (Paranaguá et al., 2016). Neste sentido, tornam-se necessários estudos relacionados com a segurança do doente na APS. A Estratégia Saúde da Família (ESF) foi o arranjo estrutural adotado pelo Ministério da Saúde no Brasil para reorientar o modelo de atençáo à saúde a partir da APS (Marchon \& Junior, 2014; Portaria no 2.436 do Ministério da Saúde, 2017). A equipa de saúde da família deve conhecer os princípios e os conceitos da segurança do doente para alicerçar a tomada de decisão no cuidado de forma mais segura, procurando práticas fundamentadas na qualidade e eficácia do cuidado (Marchon \& Junior, 2014). Assim, o presente estudo tem por objetivo analisar a perceçáo acerca da segurança do doente, de profissionais de saúde que atuam em equipas de saúde da família.

\section{Enquadramento}

A segurança do doente é uma das dimensóes da qualidade da assistência, sendo entendida como um conjunto de medidas e políticas que visam a redução, a um mínimo aceitável, do risco de ocorrência de dano desnecessário ao paciente durante a assistência (Institute of Medicine [IOM], 2000; World Health Organization [WHO], 2009). A temática destacou-se após a publicação do relatório To err is human: building a safer health system do Instituto de Medicina dos Estados Unidos da América. Este documento apresentou resultados de estudos que mostraram que os seres humanos cometem erros, os quais podem ser evitados com organização, planeamento e gestáo adequada (IOM, 2000).

Em 2013, no Brasil, foi instituído o Programa Nacional de Segurança do Paciente que propóe contribuir para a qualificaçấo do cuidado em saúde em todos os estabelecimentos de saúde do território nacional, inclusive em unidades de APS (Resoluçấo - RDC no 36 do Ministério da Saúde). Um estudo brasileiro realizado no Rio de Janeiro em unidades da ESF, constatou uma taxa de incidência de incidentes de $1,11 \%$, sendo os fatores contribuintes divididos em: falhas no cuidado $(34,00 \%)$, na gestão $(16,13 \%)$ e na comunicação $(65,53 \%$; Marchon et al., 2015). Outro estudo realizado no Brasil, em que diferentes perspetivas sobre a segurança do doente foram analisadas do ponto de vista de quem participa diariamente na APS (o gerente local, os profissionais de saúde e os pacientes), revelou barreiras para a promoção da segurança do doente, nomeadamente, a descontinui- dade dos cuidados, as interrupçóes durante as consultas, as falhas na comunicação e no trabalho em equipa. Este estudo revelou problemas de comunicação em diferentes níveis envolvendo pacientes, trabalhadores e gerentes, destacando também a importância do conhecimento compartilhado para promover a segurança do doente no cuidado primário (Vasconcelos et al., 2019).

Um estudo realizado em França constatou que os incidentes que ocorreram na APS foram três vezes mais relacionados com a organizaçáo dos cuidados de saúde do que com o conhecimento e habilidades dos profissionais de saúde e, especialmente, com o fluxo de trabalho e a comunicação entre os profissionais e com os pacientes. Constatou-se que dos 317 incidentes relatados, 270 foram considerados evitáveis (Michel et al., 2017).

Após a análise da literatura, observa-se que, de facto, acontecem incidentes na APS, sendo necessária uma melhor compreensão da segurança do doente neste importante nível de prestação de cuidados. Na verdade, um estudo realizado na Califórnia apontou ser essencial para as práticas na rede de APS, a criação de equipas que compartilham responsabilidades na assistência clínica e nas tarefas administrativas. Para isso, são necessários ambientes com estrutura física adequada e áreas comuns de trabalho entre todos os profissionais, para interação e comunicação efetiva. Por outro lado, há barreiras de implementação para a segurança da assistência na APS, como restriçóes financeiras, alta rotatividade de profissionais e resistência cultural para mudanças necessárias (Lyson et al., 2018).

\section{Questáo de investigaçáo}

Qual a perceção dos membros das equipas de saúde da família sobre a segurança do doente?

\section{Metodologia}

Estudo exploratório com abordagem qualitativa realizado em seis unidades de ESF de um município de médio porte do centro-oeste do estado de Minas Gerais, Brasil. Os participantes foram 23 profissionais que atuavam nas unidades há pelo menos 6 meses: enfermeiros, técnicos de enfermagem, médicos e agentes comunitários de saúde. Foram excluídos profissionais em período de férias ou licença de qualquer natureza. Os participantes foram identificados pelas primeiras letras referentes ao nome da sua profissão, a saber: E para enfermeiro, TE para técnico de enfermagem, $M$ para médico e AC para agente comunitário, acrescido do número de ordem das entrevistas (E1... E6; TE1... TE6; M1... M6; AC1... AC6). Os dados foram recolhidos em salas reservadas dentro das próprias unidades entre setembro de 2017 e fevereiro de 2018, através de entrevista com guiáo semiestruturado, o qual comtemplava nove questóes abertas: 1) O que você entende por segurança do doente na ESF? Qual importância desta temática neste nível de atenção? 2) O que você entende por incidente na assistência ao usuário? 3) Fale-me sobre a ocorrência e os tipos de incidentes decorrentes 
da assistência em seu quotidiano de trabalho. 4) Fale-me sobre a causa da ocorrência de incidentes decorrentes da assistência em seu quotidiano de trabalho. 5) Fale-me sobre as açôes de correção dos incidentes decorrentes da assistência em seu quotidiano de trabalho. 6) Fale-me sobre as açóes de prevençáo e minimizaçáo dos incidentes decorrentes da assistência em seu quotidiano de trabalho. 7) Como você acha que deve ser a participação do usuário na busca por sua segurança durante a assistência? Quais estratégias gestores e profissionais poderiam realizar? 8) Fale-me sobre a sala de espera como um local para promoção da segurança do doente. 9) Você já ouviu falar de um incidente que ocorreu na ESF? Você já vivenciou algum? No final, foi dada oportunidade aos participantes de abordarem algo mais dentro da temática em questáo. Ressalta-se que o guião semiestruturado foi apreciado quanto ao seu conteúdo por três docentes que atuam na área e possuem publicaçóes relativas a segurança do doente na APS.

As entrevistas foram gravadas e transcritas na íntegra para análise e categorização dos dados. A duração média das entrevistas foi de 24 minutos. Os dados foram analisados com base no referencial de Bardin (2012) em três fases: 1) pré análise: preparação, a organização e a leitura flutuante do material; 2) exploraçáo do material: que consistiu em elencar partes do texto, criar categorias para cada tipo de assunto; 3) tratamento dos resultados obtidos e interpretaçóes: elaboração de inferências nas informaçóes obtidas para serem interpretadas e utilizadas para fins teóricos ou pragmáticos.

O estudo foi aprovado pelo Comitê de Ética e Pesquisa da Universidade Federal de São João Del-Rei, sob parecer número 2.256.529, de acordo com a resolução do Conselho Nacional de Saúde 466/2012 (Resolução 466/ do Ministério da Saúde), incluindo a assinatura do termo de consentimento livre e esclarecido por todos os participantes da pesquisa.

\section{Resultados}

Dos 23 profissionais entrevistados, a maioria é do sexo feminino $(78,3 \%)$, com idade entre 27 a 55 anos, sendo que $26,1 \%$ são agentes comunitários de saúde $(50,0 \%$ com ensino médio e 50,0\% com ensino superior), 21,7\% técnicos de enfermagem, $26,1 \%$ enfermeiros e $26,1 \%$ médicos. Dos profissionais com ensino superior, $43,5 \%$ possuíam especialização em saúde da família. O tempo de atuação nas seis unidades de ESF variou entre 1 a 17 anos, sendo que o vínculo laboral de $65,2 \%$ dos participantes foi através de concurso público, e em $34,8 \%$ por meio de contratos temporários. A partir da análise dos dados, emergiram duas categorias: (Des) Conhecimento sobre os princípios da segurança do doente e Atuaçáo dos profissionais e estratégias para a promoção da segurança do doente.

Relativamente à categoria (Des) Conhecimento sobre os princípios da segurança do doente, a análise geral dos dados do estudo mostra que a segurança do doente na APS, segundo a maioria dos profissionais, é fundamental para as açôes de promoção de saúde, prevenção, tratamento e controlo de doenças e agravamento do estado de saúde. O paciente foi referido como uma personagem principal do processo do cuidado, sendo que a segurança neste nível de atenção deve ser um componente chave dos processos de trabalho, uma vez que repercute nos demais serviços. "A [APS] é a principal porta de entrada, é onde procuram o primeiro atendimento. Então se não houver uma segurança adequada aqui, o que adianta uma segurança no hospital que já é uma atenção terciária" (M4)". "Eu acho muito importante, porque o paciente é o protagonista do nosso trabalho. Então a segurança dele deveria ser o ponto principal, o ponto-chave de todo trabalho né, deveria ser o objetivo número um" (E6).

No entanto, a análise detalhada dos dados revelou que a perceção dos profissionais apresenta-se de forma diversificada. Alguns profissionais relataram aspetos importantes dos princípios de segurança do doente, mas de uma forma fragmentada e sem uma perceção ampla sobre o tema: "Quando a gente pensa em segurança do paciente já vem logo a cabeça a questáo da orientação, né, desse paciente, o direcionamento. Orientar cuidados corretos, os procedimentos corretos para sua saúde" (E1); "Entendo por segurança do paciente, que as açóes exercidas pela equipa não causam danos ao paciente" (E4).

Outros profissionais, quando se referem à segurança do doente, apresentam no discurso conceitos relacionados com as áreas correlacionadas, como a segurança do trabalhador: "Segurança do paciente é assistência correta, é tipo assim, o profissional seguro" (E3); "Eu acho que a estrutura física e de EPI [Equipamento de Proteção Individual]" (TE1).

Os enfermeiros referem conhecimento da expressão segurança do doente, perante a repercussão atual da temática, mas relatam ausência de açôes na rotina prática dos processos de trabalho da equipa de ESF, sendo que têm sido priorizadas outras açôes: "É um tema que hoje está sendo muito discutido, mas a gente foca mais em outras coisas" (E2); "Olha, é um assunto que eu tenho visto que está muito assim na mídia, e tem me chamado atenção, mas eu não conheço nenhum serviço que oferece isso" (E5). Mesmo com toda esta repercussão da segurança do doente, alguns profissionais participantes do estudo tiveram dificuldades de delimitar a abrangência da temática. Outros, por sua vez, demonstraram ausência de reflexáo a este respeito e uma estranheza quando se mencionava a segurança do doente nos dispositivos da APS: "Segurança é tudo que dá suporte ... é o suporte do paciente na Atenção Básica" (M2); "Nunca pensei sobre o assunto, mas acho que deve haver uma segurança na assistência do paciente" (M1); "É uma coisa que normalmente não se fala, por isso que estou achando até estranha a pergunta, segurança do paciente aqui dentro?” (M5).

As perceçóes dos profissionais da equipa multiprofissional das unidades de ESF evidenciam um cenário desafiador, principalmente em relação à apropriação da temática pela categoria médica. Entre outros fatores, consiste num reflexo da inexistência de açóes de segurança do doente, na vivência dos profissionais que atuam na APS e das discussōes dessa área, que está focada maioritariamente no 
contexto hospitalar: "Eu não conheço ainda a segurança do paciente na atenção primária" (E5); "Se fala muito na área hospitalar de segurança do paciente" (TE1).

É interessante observar que os profissionais percebem a lacuna existente de estudos e reflexóes sobre segurança do doente na APS, o que reverbera na prática clínica e na rotina das unidades de ESF, suscitando inclusivamente dúvidas quanto à importância da segurança do doente neste nível de prestação de cuidados: "Segurança do paciente e atenção primária é raro você ouvir falar disso. Você acha importante abordar esse assunto aqui [atenção primária à saúde]?” (TE1).

Além disso, segundo os profissionais, a prática quotidiana atual não está suportada por um planeamento prévio, que permeia açóes para a prevenção e a mitigação do erro e para a promoção da segurança do doente. No entanto, relatou-se a existência de açóes pontuais disparadas aquando da ocorrência de erros: "Não trabalha porque a gente não foca nisso. A gente atende de acordo com a demanda que vem, mas a gente nunca pensou na segurança do paciente" (AC3); "houve um erro com o usuário, a gente senta, discute e traz o erro pra toda a equipe, mas assim, são pontuais as ações. Não existe uma ação voltada só pra isso" (E5).

Por outro lado, a maioria dos participantes afirmou náo conhecer o Programa Nacional de Segurança do Paciente, instituído em 2013 pelo Ministério da Saúde. Este programa foi um marco de mudanças em prol da segurança do doente no Brasil, muito divulgado e discutido em diversos cenários da saúde no país. No entanto, alguns profissionais relataram desconhecimento total e outros alegam terem ouvido algo a respeito, mas desconhecem os seus objetivos e propostas: "Nunca ouvi falar. Já fiz pós-graduação, estudei algumas coisas de saúde da família nesses 20 anos, o programa, que eu me lembre na medicina de família, não" (M1); "Não! Nunca ouvi falar disso" (AC2); "Nunca li, mas já ouvi falar. Mas não, não conheço a fundo!" (E2).

Apesar dessa falta de conhecimento e açóes práticas no quotidiano de trabalho, chama-se a atenção que os princípios da segurança do doente estão intrínsecos no raciocínio da prática profissional, ainda que os conceitos específicos da área não sejam apropriados por estes profissionais. Houve, por exemplo, incertezas na interpretaçáo do termo "incidentes" pela maioria dos profissionais, o que pode impactar negativamente na implementação de açóes de segurança do doente nos serviços de cuidados primários à saúde: "O paciente vem pra cá pra cuidar de uma enfermidade, ele não pode adquirir outra . . . uma lesão, pode adquirir nada não, aqui dentro não" (AC5); "Incidentes na assistência ao usuário? Alguma coisa que aconteceu. . . é. . que não teve sucesso? É isso?" (E2).

Relativamente à categoria Atuação dos profissionais e estratégias para a promoção da segurança do doente, observou-se que os participantes têm uma perceção positiva da contribuição do seu trabalho individual e da equipa para a segurança do doente. No entanto, os relatos desta contribuição são genéricos, sem fundamentação teórico-científica e não traduzem as estratégias do Programa Nacional de Segurança do Paciente: "O meu trabalho?
(Silêncio). Uai eu acho que contribuí de várias formas, mas eu acho que a questão que contribui pra segurança do paciente, é fazer as condutas corretamente, é o paciente sair daqui orientado" (E1); "eu tento fazer o máximo que eu dou conta entendeu, dentro das minhas possibilidades, o que eu puder fazer para o paciente eu tento fazer da melhor forma" (TE3); "Na prevenção, no tratamento agudo e crônico. (Silêncio). Nos grupos operativos que a gente pode tá atuando, acho que é isso" (M2).

Relativamente a estratégias para a promoçáo da segurança do doente, os profissionais reconhecem e almejam açóes educacionais sobre este conteúdo. No entanto, a maioria dos profissionais relatou não ter recebido capacitação sobre segurança do doente, responsabilizando a liderança por esta ação, não se autoresponsabilizando pelo seu conhecimento: "deveria ter uma capacitação né, um treinamento de todo mundo, em equipe ... Mas treinamento relacionado à segurança do paciente, os cuidados que devem ser feito, que devem ser tomado, ainda não vi não" (TE1); "Não, não sei o que são Núcleos de Segurança. Nunca tive treinamento sobre isso, desconheço" (TE2); "eu acho que os gestores precisam capacitar mais sobre esse assunto" (AC5).

A comunicaçấo entre os profissionais da ESF foi mencionada como um ponto com necessidade de melhoria nas unidades. Ocorre de forma não sistematizada (informal), não alcança toda a equipa, além de possuir ruídos que levam a incertezas nas açóes e prejudicam os pacientes. A comunicação não efetiva foi apontada também como a causa de falhas: "sabe as vezes eu acho que é informal demais, não deveria ser né, então às vezes eu acho que fica muita falha por falta de fazer uma comunicação correta. Com certeza isso prejudica o paciente" (AC1); "Eu acho que podia ter mais comunicação, as vezes cada um toma uma atitude por conta própria, aí fica mal-entendido porque houve falta de comunicação" (TE5).

A comunicaçáo entre os profissionais e os pacientes também foi mencionada como uma estratégia para promoção da segurança do doente, sendo necessário perceber se os pacientes compreenderam o que foi transmitido pelo profissional, bem como discutir a temática nos grupos educativos dos pacientes: "é muito importante, ver que eles entenderam ... ver que consegui passar pra eles o que era importante, vendo eles fazendo tudo que foi explicado" (E3); "eu acho que em grupos educativos, às vezes poderia trabalhar com eles essa parte [segurança do paciente], né" (TE1).

Neste contexto, a sala de espera foi considerada pela maioria dos profissionais como um espaço estratégico para o aperfeiçoamento da segurança do doente por meio da mediaçấo do conhecimento sobre as questôes de saúde, corroborando também para a diminuição da ansiedade pela espera do atendimento. No entanto, a realizaçáo de processos educacionais não ocorre de forma permanente, não conta com o envolvimento de todos os membros da equipa e ainda não contempla a temática segurança do doente:

Através de cartazes eu já vi né, a residente de enfermagem fazer algumas coisas. Agora eu espero que utilizem melhor [a sala de espera], com a bi- 
blioteca que ela fez ali ... e é até uma forma de aquietar um pouco aquela angústia da espera do atendimento. (AC2)

"A gente tenta utilizar a sala de espera com bastante orientação, nós temos até o residente de enfermagem, ele às vezes faz uma programaçáo de falar todos os dias né algum assunto" (E6).

\section{Discussão}

Na primeira categoria deste estudo (Des) Conhecimento sobre os princípios da segurança do doente, observa-se que, apesar dos profissionais considerarem relevante a segurança do doente, a maioria demonstra desconhecimento e estranheza em mencionar esta temática no âmbito da APS. Numa primeira análise, pode ter-se a falsa ideia de que é desnecessário refletir sobre a segurança do doente na APS, uma vez que provavelmente os incidentes não ocorrem nestes serviços, devido à menor complexidade e à menor densidade tecnológica em relação aos restantes serviços da rede de atenção à saúde. No entanto, os incidentes na APS acontecem (Marchon et al., 2015) e todo e qualquer serviço de saúde deve ser organizado sob o princípio Primun non nocere (Primeiro não cause dano), tendo em vista que os profissionais de saúde são susceptíveis a erros.

Contudo, os estudos e as discussões sobre a segurança do doente ainda se focam no contexto hospitalar. Há uma diferença quantitativa notável de estudos referentes à segurança do doente na área hospitalar em detrimento da APS, mesmo em países com um sistema de atenção primária organizado e atuante (Marchon \& Junior, 2014). No entanto, a maioria dos cuidados de saúde são prestados na APS, incluindo as unidades de ESF, que têm um grande número de atendimentos diários (Paranaguá et al., 2016). Neste sentido, os profissionais das unidades de ESF devem compreender os princípios da segurança do doente para fundamentar a tomada de decisão, melhorando a atuação profissional e a eficácia do serviço (Marchon \& Junior, 2014).

Segundo os relatos dos participantes deste estudo, na prática quotidiana das unidades não existem açóes dirigidas à segurança do doente. As prioridades referem-se às atividades que não consideram os princípios da segurança. Dado que os procedimentos realizados na APS são aparentemente simples, as situações de riscos nos cuidados primários à saúde não recebem a devida atenção dos profissionais. É um aspeto que deve ser repensado e trabalhado pela equipa multiprofissional (Ribeiro, et al., 2017). O relatório da WHO - Cuidados de Saúde Primários - Agora Mais Que Nunca - revelou que a APS oferece cuidados pouco seguros aos pacientes, com taxas de incidentes subestimadas, sendo necessária a adoção de práticas que permitam oferecer cuidados contínuos, humanizados, com qualidade e segurança. Além disso, esse documento sublinhou que os problemas com a segurança do doente náo ocorrem apenas em hospitais, mas também com aqueles que recorrem a cuidados primários (WHO, 2008).
Dos participantes do estudo, apenas os enfermeiros destacaram nos seus discursos a repercussão atual da temática, não sendo referido por nenhum outro profissional. Além disso, verifica-se a falta de apropriação da temática pelos médicos. É um reflexo do facto de a maioria dos cursos na área da saúde não abordarem essa temática durante a formação dos profissionais (Menezes et al., 2020). Os currículos de cursos de graduação de enfermagem e medicina em países desenvolvidos já passaram por adaptaçôes. No Brasil, os erros e as falhas assistenciais sáo temas considerados como negligenciados nos cursos de enfermagem e medicina. É uma limitação que existe, porque existem diversos desafios para a mudança nos currículos nos países de baixa e média renda, como por exemplo, a pouca infraestrutura educacional, os escassos recursos financeiros e humanos, a dificuldade de integrar o conteúdo de segurança do doente com o contexto local, a falta de conhecimento das lideranças e a ausência de envolvimento e de cooperação de docentes. Apesar disso, é necessário o fortalecimento do tema da segurança do doente no ensino, na investigação e nas açóes de extensão à comunidade, instigando reflexóes importantes sobre as competências que precisam de ser desenvolvidas durante a formação académica, a fim de promover uma assistência mais segura à saúde (Menezes et al., 2020).

Os profissionais também demonstram desconhecimento sobre o Programa Nacional de Segurança do Paciente. O programa foi instituído há quase 8 anos no Brasil, sendo o marco na promoção no país de uma assistência segura à saúde. Desde então, são obrigatórias açôes estratégicas para a promoção da segurança do doente, como a instituição de Núcleos de Segurança do Paciente (NSP) nos estabelecimentos de saúde. O NSP tem por objetivo promover e apoiar a implementaçáo de açóes voltadas para a segurança do doente, sendo essencial para a busca pela qualidade das atividades desenvolvidas na APS (Resolução - RDC no 36 do Ministério da Saúde).

Os profissionais também demonstraram dúvidas quanto a conceitos importantes, como incidente. $\mathrm{O}$ termo "incidente" é definido como um evento ou circunstância que poderia resultar, ou resultou, em dano desnecessário para o paciente e originam-se de atos intencionais dos profissionais (violaçóes) ou de atos não intencionais (erros). Um erro é a falha na execução de uma ação planeada de acordo com o desejado ou o desenvolvimento incorreto de um plano. O evento adverso é aquele incidente que causou um dano decorrente da prestação de cuidados de saúde. Um dano inclui doenças, lesão, sofrimento, incapacidade ou disfunção e morte. $\mathrm{O}$ dano pode, assim, ser físico, social ou psicológico (WHO, 2009). A Classificação Internacional de Segurança do Doente (International Classification for Patient Safety - ICPS) da WHO aborda todos estes conceitos, abarcando os principais termos da área. Faz-se necessário que os profissionais da saúde conheçam os termos e as definições para identificação, investigação, mitigação e prevenção de incidentes.

A segunda categoria Atuação dos profissionais e estratégias para a promoçáo da segurança do doente mostra a percepção dos profissionais quanto à importância da participação, individual e da equipa multiprofissional, 
no cuidado, bem como algumas melhorias necessárias para a promoção da segurança do doente no quotidiano de trabalho das unidades de ESF.

Verificam-se dificuldades dos profissionais em relatar como o seu trabalho contribui para a promoção de uma assistência segura, reafirmando o baixo conhecimento quanto ao tema discutido. É preciso que os profissionais, no seu quotidiano de trabalho, realizem um cuidado pautado na segurança dos pacientes, mas para isso precisam de conhecer o tema e reconhecer o seu papel neste processo. Por outro lado, é importante que a liderança dos serviços de saúde implemente açôes focadas nos princípios da segurança do doente, como uma comunicação efetiva, estímulos à promoção do desenvolvimento profissional por meio de capacitaçóes, realização de incentivos às práticas seguras e realizaçáo de feedback para o profissional (Reis et al., 2017). Os participantes afirmaram não ter açóes educacionais focadas na segurança do doente. A educação permanente para profissionais do Sistema Único de Saúde tem sido vista como instrumento para transformaçōes das práticas profissionais e da organização do trabalho. A capacitaçáo ou a formação sobre segurança do doente são essenciais, e devem ser realizadas durante o período da jornada de trabalho, abordando assuntos como legislaçóes e princípios da ciência de segurança do doente; protocolos, indicadores e cultura de segurança do doente; gestáo de riscos; investigação e tratamento de incidentes, análise de causa-raiz e estratégias para a melhoria da segurança.

No entanto, chama a atenção o facto de os profissionais responsabilizarem somente os gestores por esta ação e não se autoresponsabilizarem em aprender esse conteúdo importante. A equipa tem como missão e desafio prezar e propor estratégias para o cuidado e prestação de cuidados de boa qualidade de forma a criar estratégias para impedir a prestaçáo de cuidados de saúde inadequados e a ocorrência de complicaçóes evitáveis (Ribeiro et al., 2017).

A comunicaçáo entre os profissionais é vista também como mecanismo de colaboração para a promoção da segurança do doente nas unidades de ESF. No entanto, a comunicação entre os profissionais apresenta grandes lacunas, o que pode levar a falhas no cuidado. Alguns estudos demonstram que a comunicação não efetiva entre os profissionais de saúde, tem sido um dos principais fatores que dá origem a incidentes, afetando a qualidade do cuidado prestado (Lee, 2015; Lawson et al., 2018). Assim, como a comunicação entre profissionais de saúde ocorre em diferentes lugares e situações, é necessário padronizá-la e sistematizála em todos os níveis hierárquicos e organizacionais de uma instituição (Lee, 2015).

Por fim, a sala de espera foi apontada como um ambiente estratégico para mediar o conhecimento sobre as questôes de saúde, inclusive no que concerne à segurança do doente. A educação para a saúde na sala de espera é uma importante ferramenta de trabalho quando utilizada como meio de acolhimento, de comunicabilidade e de problematização da realidade. A partir da prática de educação em saúde é possível desenvolver a troca de conhecimento, de experiências, onde o saber profissional e o bom senso se unem em benefício do senso comum, resultando na construção de indivíduos mais críticos e conscientes (Wild et al., 2014).

Perante o contexto apresentado, pode inferir-se que as unidades de ESF estudadas ainda não prestam um cuidado primário baseado nos princípios da segurança do doente, por escassez de informação. Assim, é necessário colocar em prática as estratégias mencionadas pelos participantes, de forma a prevenir e mitigar os riscos provenientes de uma assistência insegura.

A limitaçáo da pesquisa refere-se à realização em apenas seis unidades de ESF, o que restringe a generalização dos resultados. Assim, faz-se necessário ampliar o estudo que abarque outras realidades da APS, além de investigar os impactos das estratégias de promoção da segurança do paciente na APS na rede de atenção à saúde.

\section{Conclusão}

A segurança do doente na APS ainda é pouco reconhecida e valorizada entre os profissionais do estudo. Evidenciou-se estranheza e lacunas no conhecimento sobre a temática, o que pode gerar consequências negativas na assistência à saúde ao paciente, com maior risco de ocorrência de incidentes.

Destaca-se que apenas os enfermeiros destaquem a repercussão atual da temática e que a categoria médica não demonstre apropriação sobre os princípios da segurança do doente. Assim, faz-se necessário capacitar os profissionais da APS sobre a segurança do doente, como também incentivá-los à apropriação individual dos princípios desta. Para além disso, é necessário incluir o tema nos currículos de graduação dos profissionais da área da saúde.

Por fim, o movimento de melhoria contínua da qualidade da assistência e da segurança do doente deve contemplar todos os profissionais de saúde da APS com vista a desenvolver uma cultura direccionada para a promoção da segurança do doente.

\section{Contribuição de autores}

Conceptualização: Ribeiro, H. C., Pardini, R. D., Franco, E. C.

Tratamento de dados: Ribeiro, H. C., Pardini, R. D.

Análise formal: Ribeiro, H. C., Pardini, R. D., Silva, J.

Investigação: Ribeiro, H. C., Pardini, R. D.

Metodologia: Ribeiro, H. C., Pardini, R. D.

Administração do projeto: Ribeiro, H. C..

Recursos: Ribeiro, H. C., Pardini, R. D.

Supervisão: Ribeiro, H. C., Silva, J.

Validação: Ribeiro, H. C., Pardini, R. D., Silva, J., Menezes, A. C., Franco, E. C., Mata, L. R., Campos, C. G., Visualização: Ribeiro, H. C., Pardini, R. D.

Redação - rascunho original: Pardini, R. D., Ribeiro, H. C. Redação - análise e edição: Ribeiro, H. C., Pardini, R. D., Silva, J., Menezes, A. C., Franco, E. C., Mata, L. R., Campos, C. G. 


\section{Referências bibliográficas}

Bardin, L. (2012). Análise de Conteúdo. Ediçôes 70.

Institute Of Medicine. (2000). To err is human: Building a safer health system. National Academies Press.

Lawson, S., Reid, J., Morrow, M., \& Gardiner, K. (2018). Simulation-based education and human factors training in postgraduate medical education: A Northern Ireland perspective. The Ulster Medical Journal, 87(3), 163-167.

Lee, J. Y. (2015). Effective communication for patient safety. Journal of the Korean Medical Association, 58(2),100-104. http://dx.doi. org/10.5124/jkma.2015.58.2.100

Lyson, H. C., Ackerman, S., Lyles, C., Schillinger, D., Williams, P., Gourley, G., Sarkar, U. (2018). Redesigning primary care in the safety net: A qualitative analysis of team-based care implementation. Healthcare, 9(4), 1- 8. https://doi.org/10.1016/j. hjdsi.2018.09.004

Marchon, S. G., Junior, W. V., \& Pavão, A. L. (2015). Características dos eventos adversos na atençáo primária à saúde no Brasil. Caderno de Saúde Pública, 31(11), 2313-2330. https://doi.org/10.1590/ 0102-311X00194214

Marchon, S. G., \& Junior, W. V. (2014). Segurança do paciente na atenção primária à saúde: Revisão sistemática. Caderno de Saúde Pública, 30(9), 1-21. http://dx.doi.org/10.1590/0102-311X00114113

Menezes, A. C., Penha, C. S., Amaral, F. M., Pimenta, A. M., Ribeiro, H. C., Pagano, A. S., \& Mata, L. R. (2020). Latino Students Patient Safety Questionnaire: Adaptação transcultural para estudantes brasileiros de enfermagem e de medicina. Revista Brasileira de Enfermagem, 73(Supl. 6), 1-8. http://dx.doi.org/10.1590/00347167-2019-0621

Michel, P., Brami, J., Chanelière, M., Kret, M., Mosnier, A., Dupie, I., Haeringer-Cholet, A., Keriel-Gascou, M., Maradan, C., Villebrun, F., Makeham, M., \& Quenon, J. L. (2017). Patient safety incidents are common in primary care: A national prospective active incident reporting survey. Plos One, 12(2). https://doi. org/10.1371/journal.pone.0165455
Paranaguá, T. T., Bezerra, A. L., Tobias, G. C., \& Ciosak, S. I. (2016). Suporte para aprendizagem na perspectiva da segurança do paciente na atenção primária em saúde. Revista Latino-Americana de Enfermagem, 24, 1-9. https://doi.org/10.1590/1518-8345.0784.2771

Portaria no 2.436, do Ministério da Saúde. (2017). Diário Oficial. https://bvsms.saude.gov.br/bvs/saudelegis/gm/2017/ prt2436_22_09_2017.html

Ribeiro, H. C., Montenegro, L. C., Mata, L. R., \& Marchon, S. G. (2017). Qualidade e segurança no cuidado na atenção primária à saúde. Guanabara Koogan.

Resolução no 466 do Ministério da Saúde. Diário Oficial. https://bvsms. saude.gov.br/bvs/saudelegis/cns/2013/res0466_12_12_2012.html

Resolução - RDC no 36 do Ministério da Saúde. Diário Oficial. http://bvsms.saude.gov.br/bvs/saudelegis/anvisa/2013/ rdc0036_25_07_2013.html

Reis, G. A., Hayakawa, L. Y., Murassaki, A. C., Matsuda, L. M., Gabriel, C. S., \& Oliveira, M. L. (2017). Implantaçáo das estratégias de segurança do paciente: Percepçóes de Enfermeiros Gestores. Texto Contexto Enfermagem, 26(2), 1-9. https://doi. org/10.1590/0104-07072017000340016

Vasconcelos, P. F., Freitas, C. H., Jorge, M. S., Carvalho, R. E., Freire, V. E., Araújo, M. F., Aguiar, M. I., Oliveira, G. Y., Anjos, J. S., \& Oliveira, A. C. (2019). Safety attributes in primary care: Understanding the needs of patients, health professionals, and managers. Public Health, 17(1), 31-40. https://doi.org/10.1016/j. puhe.2019.03.021

Wild, C. F., Silveira, A., Favero, N. B., Rosa, E. O., Gueterres, E. C., \& Leal, S. D. (2014). Educação em saúde na sala de espera de uma policlínica infantil: Relato de experiência. Revista de Enfermagem da UFSM, 4(3), 660-666. https://doi.org/10.5902/2179769212397

World Health Organization. (2008). Cuidados de saúde primários: Agora mais que nunca. https://www.who.int/eportuguese/publications/whr08_pr.pdf

World Health Organization. (2009). Conceptual framework for the international classification for patient safety. https://www.who.int/ patientsafety/implementation/taxonomy/ICPS-report/en 
Ribeiro, H. C. et al. 